\title{
A PROPERTY OF CONTINUA SIMILAR TO LOCAL CONNECTIVITY*
}

\author{
BY W. A. WILSON
}

1. Introduction. An important property of locally connected compact spaces is that the complement of every continuum is the sum of a finite or enumerably infinite set of connected regions. If, however, a compact space $Z$ is not locally connected and $X$ is a sub-continuum, neither of the properties of $Z$ being locally connected about $X$ and $Z-X$ being the sum of an at most enumerably infinite set of connected regions involves the other. A property that is stronger than that of $Z$ being locally connected about $X$ is the following, introduced in a recent paper $\dagger$ by G. T. Whyburn: A sub-continuum $X$ is $\epsilon$-separated by a finite set of sub-continua of $Z$ if, for each $\epsilon>0$, there is a finite set of sub-continua $\left\{F_{i}\right\}$ of $Z$ such that $Z-\sum_{1}^{m} F_{i}$ is the sum of two separated sets $Z_{1}$ and $Z_{2}$, where $Z_{1}$ contains $X$ and all points of $Z_{1}+\sum_{1}^{m} F_{i}$ have a distance less than $\epsilon$ from $X$. It is the purpose of this paper to describe another quasi-local property and its relation to those just mentioned, which in strength lies between local connectivity and $\epsilon$-separability. The work will be carried out for separable metric continuous spaces in which the Bolzano-Weierstrass property is valid; such spaces will be called $W$-spaces for the sake of brevity.

Definitions. If $a$ and $b$ are disjoint bounded continua (or points) in a $W$-space $Z$, and $Z$ can be expressed as the sum of two continua $H$ and $K$ such that $H \cdot b=K \cdot a=0$, we say that $Z$ is divisible between $a$ and $b$.

If $Z$ is divisible between the bounded sub-continuum (or point) $x$ and every sub-continuum of $Z-x$, then $x$ is called biregular.

For example, let $Z$ consist of a segment $a b$ of unit length and an enumerable set of segments $a b_{n}$, each of unit length and inclined to $a b$ at the angle $\pi / n$. Then $Z$ is locally connected at $a$,

* Presented to the Society, September 9, 1930.

$\dagger A$ generalized notion of accessibility, Fundamenta Mathematicae, vol. 14, pp. 311-326. 
or, as it is sometimes stated, $a$ is a regular point, but $a$ is not biregular, nor is it $\epsilon$-separable, nor is its complement the sum of connected regions. However, $a b$ has all these properties.

A condition weaker than biregularity is for $Z$ to be divisible between $x$ and every point of $Z-x$. Thus, if $Z$ consists of a unit segment $a b$ and an enumerable set of arcs $\left\{C_{n}\right\}$, each terminated by $a$ and $b$ and of radius $n$, and $x$ is a point of $a b-(a+b)$, $Z$ is divisible between $x$ and every point of $Z-x$, but $x$ is neither regular nor biregular, although its complement is a semi-continuum. Since in this example the point $a$ is a biregular continuum, we see that biregularity is not, strictly speaking, a local property.

2. Lemma. Let $Z$ be a connected $W$-space, $x$ be a bounded subcontinuum, and $Z$ be divisible between $x$ and every point of $Z-x$. Then for each $\epsilon>0$, there is a least integer $m$ such that the set of points of $Z$ whose distances from $x$ are not less than $\epsilon$ can be covered by $m$ disjoint sub-continua of $Z-x$.

PROof. Here and elsewhere we denote the set of points of $Z$ whose distances from $x$ are less than $\epsilon$ by $V_{\epsilon}(x)$. If $y$ is any point of $Z-V_{\epsilon}(x)$, we have $Z=H_{y}+K_{y}$, where $H_{y}$ and $K_{y}$ are continua and $y \cdot H_{y}=x \cdot K_{y}=0$. Let $F$ be the frontier of $V_{\epsilon}(x)$. Then $F$ is a bounded closed set and each of its points is an inner point of some $K_{y}$. By the Borel theorem, $F$ is covered by a finite set of the continua $\left\{K_{y}\right\}$, say $K_{1}, K_{2}, \cdots, K_{n}$.

The set $T=Z-V_{\epsilon}(x)+\sum_{1}^{n} K_{i}$ is a closed set and each point of $F$ is an inner point of some $K_{i}$. Hence $F$ is covered by some finite set of components of $T$, say $C_{1}, C_{2}, \cdots, C_{r}$. Unless $r=1$, $T$ is not connected; suppose that it can be decomposed into $s$ mutually disjoint closed sets $\left\{T_{i}\right\}$. If $s>r$, some $T_{i}$, say $T_{s}$, would contain no $C_{i}$ and $Z$ would be the sum of two disjoint closed sets, $T_{s}$ and $V_{\epsilon}(x)+\sum_{1}^{s-1} T_{i}$, a contradiction. Thus $s \leqq r$ and we can take $s=r$, since there are $r$ components $\left\{C_{i}\right\}$. It follows, then, that each $T_{i}=C_{i}$ and so $Z-V_{\epsilon}(x)$ can be covered by $r$ disjoint continua.

As $r$ is finite, there is some least value of $r$ which we take as $m$.

3. Theorem 1. Let $Z$ be a connected $W$-space and $x$ be a biregular continuum. Then $Z$ is locally connected about $x$. 
In a previous note* it was shown that, if $Z$ is divisible between every pair of disjoint sub-continua, then $Z$ is everywhere locally connected. The demonstration of the above theorem is nearly the same.

By the lemma of the previous section we know that for each $\epsilon>0$ there is a least integer $m$ such that $Z-V_{\epsilon}(x)$ is covered by $m$ disjoint continua $\left\{S_{i}\right\}$, none of which contains any point of $x$. From this point on the proof in the reference given, beginning at the top of page 87 , is applicable almost verbatim, since $Z$ is divisible between $x$ and every sub-continuum of $Z-x$.

This theorem together with the first example in $\$ 1$ shows that the condition of biregularity is stronger than that of local connectivity. However, if $Z$ is locally connected about the bounded continuum $x$ and also $Z$ is divisible between $x$ and every point of $Z-x$, then $x$ is biregular. For, if $y$ is a sub-continuum of $Z-x$ and $\epsilon$ is less than the distance between $x$ and $y$, there is a continuum $X$ such that some $V_{\delta}(x) \subset X$ and $X \subset V_{\epsilon}(x)$. By $\$ 2$, $Z-V_{\delta}(x)$ is covered by $m$ disjoint continua $\left\{S_{i}\right\}$, none of which meets $x$. If $y \subset S_{1}$, then $X^{\prime}=X+\sum_{2}^{m} S_{i}$ is a continuum, $Z=X^{\prime}$ $+S_{1}, y \cdot X^{\prime}=0$, and $x \cdot S_{1}=0$. Hence $x$ is biregular.

4. Theorem 2. Let $Z$ be a connected $W$-space, $x$ be a bounded sub-continuum, and $Z$ be divisible between $x$ and every point of $Z-x$. Then $Z-x$ has a finite or enumerable set of components and each component is both a region and a semi-continuum.

Proof. Take $\epsilon_{1}>0$. By $\$ 2$ we know that $Z-V_{\epsilon_{1}}(x)$ is covered by $m_{1}$, but not less than $m_{1}$, mutually disjoint continua $\left\{K_{r}^{1}\right\}, r=1,2, \cdots, m_{1}$, no one of which contains a point of $x$. Take $\epsilon_{2}$ less than half $\epsilon_{1}$ and less than the least distance between $x$ and any $K_{r}^{1}$. Then $Z-V_{\epsilon_{2}}(x)$ is covered by $m_{2}$, but not less than $m_{2}$, mutually disjoint continua $\left\{K_{r}^{2}\right\}, r=1,2, \cdots, m_{2}$, no one of which contains a point of $x$. As $K_{1}^{1} \subset Z-V_{\epsilon_{2}}(x), K_{1}^{1}$ is an inner set of some $K_{r}^{2}$, say $K_{1}^{2}$. No $K_{r}^{1} \subset K_{1}^{2}$ if $r \neq 1$, for then $Z-V_{\epsilon_{1}}(x)$ would be covered by $m_{1}-1$ continua. Let, then, $K_{1}^{1} \subset K_{1}^{2}, K_{2}^{1} \subset K_{2}^{2}, K_{3}^{1} \subset K_{3}^{2}$, etc. We note that $m_{1} \leqq m_{2}$.

Now take $\epsilon_{3}$ less than half $\epsilon_{2}$ and less than the least distance between $x$ and any $K_{r}{ }^{2}$ and define the continua $\left\{K_{r}^{3}\right\}, r=1$,

* A property of continua equivalent to local connectivity, this Bulletin, vol. 36, pp. 85-88. 
$2, \cdots, m_{3}$, as before. Continue this process indefinitely. We then have

$$
\begin{aligned}
& K_{1}{ }^{1} \subset K_{1}{ }^{2} \subset K_{1}{ }^{3} \subset \cdots, \\
& K_{2}{ }^{1} \subset K_{2}{ }^{2} \subset K_{2}{ }^{3} \subset \cdots, \\
& K_{3}{ }^{1} \subset K_{3}{ }^{2} \subset K_{3}{ }^{3} \subset \cdots
\end{aligned}
$$

For each $r$ let $L_{r}$ be the union of the sequence of continua $\left\{K_{r}{ }^{i}\right\}$. Then each $L_{r}$ is a semi-continuum and, since $\epsilon_{i} \rightarrow 0, Z-x$ is the union of the semi-continua $\left\{L_{r}\right\}$. If, from some $i$ on, we have $m_{i}=n$, there are $n$ semi-continua $\left\{L_{r}\right\}$; if $m_{i} \rightarrow \infty$ as $\epsilon_{i} \rightarrow 0$, there is an enumerable set of the semi-continua $\left\{L_{r}\right\}$.

We first note that $L_{r} \cdot L_{s}=0$, if $r \neq s$. For in the case of every $i, K_{r}{ }^{i} \cdot K_{s}{ }^{i}=0$ if $r \neq s$. We now show that each $L_{r}$ is a region. If $y$ is any point of $L_{r}, Z=H+K$, where $H$ and $K$ are continua and $x \cdot K=y \cdot H=0$. Then for some $i, K \cdot V_{\epsilon_{i}}(x)=0$, whence $K$ lies in some $K_{r}{ }^{i}$; that is, $K \subset L_{r}$. But $y$ is an inner point of $K$, and consequently of $L_{r}$. Finally, since the sets $\left\{L_{r}\right\}$ are disjoint connected regions, they are components of $Z-x$. Thus the theorem is proved.

Note. The theorem is valid a fortiori if $x$ is biregular. The proof also shows that each component of $Z-x$ is the union of an ascending sequence of continua, each of which is an inner set of those following. The converse is not true, as is seen from the first example in $\$ 1$, but we do have the following as a sort of converse.

Theorem 3. Let $Z$ be a connected compact space, $x$ be a subcontinuum, $Z$ be locally connected about $x$, and every component of $Z-x$ be a region and a semi-continuum which is the union of an ascending sequence of continua such that each one is an inner set of the following. Then $x$ is biregular.

Proof. Let $R$ be any component of $Z-x, S$ be the sum of the others, and $y$ be a point of $R$. Then $y$ is an inner point of some sub-continuum $K$ of $R$. Let $\epsilon$ be half the distance between $x$ and $K$. Since $Z$ is locally connected about $x$, there is a $\delta>0$ and a continuum $X$ such that $V_{\delta}(x) \subset X \subset V_{\epsilon}(x)$ and $K \cdot X=0$.

Every component of $\bar{R}-(X+K)$ has limiting points on $X$ or $K$, but none on $x$ or $y$. Let $L$ and $M$ be the sum of $X$ and $K$, respectively, and the components of $\bar{R}-(X+K)$ having limiting points on $X$ and $K$, respectively. Then $\bar{L}+S$ and $\bar{M}$ are 
continua and $Z=(\bar{L}+S)+\bar{M}$ is a division of $Z$ between $x$ and $y$. Since $Z$ is locally connected about $x$ and is divisible between $x$ and every point of $Z-x, x$ is biregular.

Simple examples show that the theorem may fail if any of the hypotheses are omitted.

5. Theorem 4. Let $Z$ be a connected compact space, $x$ be a biregular sub-continuum, and $R_{i}$ be any component of $Z-x$. If $M_{i}=x+R_{i}$, each $M_{i}$ and the union of any number of the $M_{i}$ are biregular continua. Also $x$ is biregular with respect to each $M_{i}$.

Proof. Let $A$ be the union of any number of the continua $\left\{M_{i}\right\}$ and let $y$ be any sub-continuum of $Z-A$. Now $A$ is a continuum, because it is connected and $Z-A$ is the sum of a finite or enumerable set of disjoint regions. The continuum $y$ lies in some $R_{i}$, say in $R_{1}$, and $Z=H+K$, where $H$ and $K$ are continua and $x \cdot K=y \cdot H=0$. As $x \cdot K=0, K \subset R_{1}$ and $A \subset H$; hence $A \cdot K=y \cdot H=0$. Thus $A$ is biregular.

Now consider any one of the continua $\left\{M_{i}\right\}$, say $M_{1}$. If $y$ is a sub-continuum of $M_{1}-x$, it lies in $R_{1}$. Then $K \subset R_{1}$ and $x \subset H$. As $x$ is bounded, each component of $H-x$ has limiting points on $x$; hence $H_{1}=H \cdot M_{1}=x+H \cdot R_{1}$ is a continuum. Thus $M_{1}$ is the union of two continua $K$ and $H_{1}, y \cdot H_{1}=0$, and $x \cdot K=0$, which was to be proved.

6. Theorem 5. Let $Z$ be a connected $W$-space and $x$ a biregular sub-continuum. Then for every $\epsilon>0$ there is a least integer $m$ such that $Z$ is the union of a continuum $X$ containing some $V_{\delta}(x)$ and contained in $V_{\epsilon}(x)$, and $m$ mutually disjoint continua $\left\{K_{i}\right\}$, each of which contains no point of $x$.

Proof. By $\S 3$ there is a continuum $X$ such that some $V_{\delta}(x) \subset X \subset V_{\epsilon}(x)$. By $\$ 2$ there are $m$ mutually disjoint continua $\left\{K_{i}\right\}$ such that $Z-V_{\delta}(x) \subset \sum_{1}^{m} K_{i}$ and $x \cdot K_{i}=0$ for each value of $i$. As $m$ is finite, there is clearly some choice of $X$ and the $K_{i}$ so that $m$ has a least value.

CoROLlary. If in addition to the above hypotheses $Z$ is at most $n$-coherent, ${ }^{*}$ then $x$ is $\epsilon$-separable by a finite set of continua.

* This term signifies that for every partition of $Z$ into two continua $H$ and $K, H \cdot K$ has not more than $n$ components. This mode of classifying continua is a modification of a definition by C. Kuratowski. 
For $X \cdot K_{i}$ has at most $n$ components for each value of $i$ and so $X \cdot \sum_{1}^{m} K_{i}$ has at most $m n$ components.

As remarked in $\$ 4$, the integer $m$ may increase indefinitely as $\epsilon \rightarrow 0$, or it may remain bounded. Generalizing Menger's notion of order of a point, we may say in the former case that $x$ is of order $w$; in the latter, of finite order. If $Z$ is unicoherent, the order of $x$ is $w$ if $Z-x$ has an infinite set of components, and is the same as the number of components of $Z-x$ if that number is finite. This corollary shows that for at most $n$-coherent continua biregularity is a local property.

7. Theorem 6. Let $Z$ be a connected $W$-space, $x$ be a bounded sub-continuum, and $x$ be $\epsilon$-separated by a finite set of continua. Then $x$ is biregular.

Proof. Let $y$ be any sub-continuum of $Z-x$ and $\epsilon$ be half the distance between $x$ and $y$. By hypothesis there is a region $R$ such that $x \subset R \subset V_{\epsilon}(x)$ and $F$, the frontier of $R$, is contained in the sum of $m$ bounded sub-continua $\left\{F_{i}\right\}$ of $V_{\epsilon}(x)$.

Let $G=Z-R$. The closed set $G$ cannot be decomposed into more than $m$ mutually disjoint closed sets $\left\{G_{i}\right\}$, for, if this were possible, one of them, say $G_{1}$, would contain no point of $F$ and we would have $Z$ decomposed into two disjoint closed sets, $G_{1}$ and $Z-G_{1}$. Hence $G$ is the sum of $r$ mutually disjoint continua $\left\{G_{i}\right\}$, and $r \leqq m$.

Similarly, since every component of $R$ has limiting points on at least one $F_{i}, \bar{R}$ can be decomposed into $k$ mutually disjoint continua $\left\{C_{i}\right\}$, where $k \leqq m$, and one of them, say $C_{1}$, contains some $V_{\delta}(x)$. Clearly $G+\sum_{2}^{k} C_{i}$ is the sum of a finite number of mutually disjoint continua $\left\{K_{j}\right\}$, none of which contains a point of $x$, all of which meet $C_{1}$, and one of which, say $K_{1}$, contains $y$. Let $H_{1}$ be the union of $C_{1}$ and the other continua $\left\{K_{j}\right\}$. Then $Z=H_{1}+K_{1}, y \cdot H_{1}=0$, and $x \cdot K_{1}=0$. Hence the theorem is proved.

8. Compact Space. If $Z$ is a bounded $W$-space, that is, a compact space, $Z$ itself formally satisfies the definition of biregularity. It is easily seen from the definition that the divisor of a descending sequence of biregular continua is biregular; hence each point $x$ lies on a continuum $X$ irreducible with respect to the properties of being biregular and containing $x$. 
If there is an upper semi-continuous decomposition of $Z$ into sub-continua about each of which $Z$ is locally connected, it is clear that each element is biregular. It would then appear that at least in the case of at most $n$-coherent continua the irreducibly biregular sub-continua would serve as elements of such a decomposition. Unfortunately they need not be unique. However, one special case is not without interest.

Theorem 7. Let $Z$ be a connected compact space and let $Z$ be divisible between no two points of any irreducibly biregular subcontinuum. Then there is an upper semi-continuous decomposition of $Z$ into elements $\{X\}$, which are the irreducibly biregular subcontinua of $Z$.

Proof. We first note that each point of $Z$ lies on only one irreducibly biregular sub-continuum. For, if a point $x$ were on two such, say $X$ and $X^{\prime}$, and $y$ were a point of $X^{\prime}$ not on $X$, a division of $Z$ between $X$ and $y$ would be a division between two points of $X^{\prime}$.

Now let $\left\{X_{i}\right\}$ be a sequence of elements and $X$ be an element such that, for $\epsilon>0$ and every $i$ larger than some $i^{\prime}, X_{i} \cdot V_{\epsilon}(X) \neq 0$. If $Z-X$ contains a point of the upper closed limiting set $X^{\prime}$ of the sequence $\left\{X_{i}\right\}$, we can assume that for a partial sequence each $X_{i}$ contains two points $x_{i}$ and $y_{i}$ such that $x_{i} \rightarrow a$ and $y_{i} \rightarrow b$, where $a$ lies in $X$ and $b$ in $Z-X$. As $Z$ is divisible between $X$ and $b$, and so between $a$ and $b$, there is an $i$ so large that $Z$ is divisible between $x_{i}$ and $y_{i}$, a contradiction. Since $Z$ is locally connected about each $X$, the theorem is proved.

In this special case the decomposition is the finest possible, for some elements of a finer decomposition would be biregular subcontinua and proper sub-continua of some $X$, an impossibility. If there is a region composed of biregular sub-continua $\{X\}$ such that $Z$ is divisible between no two points of any $X$, we can obtain an upper semi-continuous decomposition analogous to that into prime parts by taking the components of the remainder of $Z$ as elements.

YaLe University 